\title{
Utility of fine needle aspiration cytology (FNAC) in the diagnosis of soft tissue tumors and tumor like lesions
}

\author{
Anitha Padmanabhan ${ }^{1, *}$, Sonali Rajesh Saraf ${ }^{2}$, Vandita Singh ${ }^{3}$, Nikita Anil Patel ${ }^{4}$ \\ ${ }^{1-3}$ Assistant Professor, ${ }^{4}$ Resident, Dept. of Pathology, LTMMC \& LTMGH Sion Mumbai, Maharashtra, India
}

*Corresponding Author:

Email: anithahari2001@ hotmail.com

Received: $31^{\text {st }}$ March, 2016

Accepted: $04^{\text {th }}$ December, 2017

\begin{abstract}
Introduction: Fine needle aspiration cytology (FNAC) forms one of the first diagnostic tools in evaluation of soft tissue lesions. Aim of this study was to evaluate the role of FNAC in the primary diagnosis of soft tissue tumors and tumor like lesions and to assess the effectiveness of FNAC in the histological subtyping and grading of soft tissue tumors.

Materials and Methods: We reviewed FNAC smears of 170 soft tissue tumors and tumor like lesions over a 3 years period and correlated with the histopathological features which were available in 117 cases. Conventional Papanicolaou (PAP) and Giemsa staining was done in all cases.

Results: 152 out of 170 cases [89.41\%] were neoplastic, of which 130 tumors were labelled as benign. Lipoma constituted $83.07 \%$ of benign tumors. Based on the predominant cytomorphological features and background, the tumors were classified into myxoid (4), spindle cell (20), pleomorphic (2), small round cell(4), epithelioid /polygonal cell (7), lipomatous (108) and miscellaneous (7). The non-neoplastic lesions included tuberculosis, filariasis, actinomycosis, scar endometriosis and calcinosis cutis.

Conclusion: FNAC proved to be helpful in distinguishing between neoplastic and non-neoplastic soft tissue lesions, differentiating metastatic carcinoma and melanoma in soft tissue from primary soft tissue tumors and differentiating benign and malignant soft tissue tumors. Accurate subtyping was possible in many tumors of myxoid category and round cell tumors. Spindle cell tumors of intermediate grade were difficult to grade on FNAC. Pleomorphic sarcomas were of high grade and their subtyping could not be done on FNAC.
\end{abstract}

Keywords: FNAC, Soft tissue lesions, Soft tissue tumors.

\section{Introduction}

Soft tissues are the supportive tissue of various organs as well as the nonepithelial, extraskeletal structures. They include adipose tissue, fibrous connective tissue, skeletal muscle, blood vessels, and the peripheral nervous system. They are almost entirely derived from the mesoderm except for the peripheral nerves. Soft tissue tumors (STTs) are a highly heterogeneous group of tumors and are classified on a histogenetic basis according to their resemblance to adult tissue. ${ }^{1}$ Primary role of cytopathologists in FNAC of soft tissue lesions is to determine whether the lesion is neoplastic or reactive and if neoplastic, whether it is benign or malignant.

Since the therapy of sarcoma depends on histological subtype, grade, stage and anatomic location, establishing a specific histological subtype and suggesting the grade is important in the FNAC report. The incidence of benign soft tissue tumors is about ten times that of malignant ones. ${ }^{1}$ Benign deep masses in adults are usually due to intramuscular lipoma. Extremity masses larger than $5-7 \mathrm{~cm}$ and deeper than subcutaneous tissue, favour the diagnosis of a malignant soft tissue tumor. Benign tumors are usually superficial and well defined or encapsulated masses showing slow growth. Of the imaging methods commonly used for evaluation, magnetic resonance imaging best defines the relationship between a tumor and its adjacent anatomic structures, such as compartment boundaries, nerves, vessels, and muscle. ${ }^{2}$

We describe our experience with the use of FNAC as an initial diagnostic procedure in cases of soft tissue lesions.

\section{Materials and Methods}

We did a retrospective study at a tertiary care hospital and reviewed FNAC smears of 170 cases over a 3 year period. Histopathological correlation was obtained in 117 cases of which 114 were resected specimens and 3 were biopsies. FNAC was performed manually in 168 cases using 23 gauge needles while CT guided aspirates were received in 2 cases. Per case 4 slides were were prepared. We performed (MayGrunwald Giemsa) MGG stain on the air dried smears ( 2 slides). While 2 smears were fixed with hair spray (denatured alcohol) and subsequently stained with Papanicolaou stain. The histopathological correlation was done on H\&E stained sections from the resected specimens and biopsies. Haemorrhagic aspirates and smears with other obscuring factors were labeled as inadequate smears and were excluded from our study. Immunohistochemistry (IHC) however diagnostic it could not be performed as there was lack of setup for the required IHC panel at our centre. 


\section{Results}

In the present study, we divided the cases into neoplastic and non-neoplastic. The neoplastic lesions were further classified into 7 separate groups based on the predominant cytomorphologic features. The sub groups included- myxoid $(n=4)$, spindle cell $(n=20)$, pleomorphic $(n=2)$, small round cell $(n=4)$, epithelioid /polygonal cell $(\mathrm{n}=7)$, lipomatous $(\mathrm{n}=108)$, miscellaneous $(n=7)$.

Table 1 gives the distribution of the cases in myxoid category. The main feature in this myxoid category $(n=4)$ is the presence of voluminous extracellular matrix material seen as irregularly shaped fragments with or without embedded tumor cells. With MGG, they appear reddish purple. Smears from CT guided aspirate from a retroperitoneal mass showed extracellular myxoid material, branching capillaries and lipoblasts. The presence of lipoblasts helped us in giving a definite diagnosis of myxoid liposarcoma which was confirmed on histology.

The cells of myxofibrosarcoma showed moderate pleomorphism and presence of spindle cells also. Since lipoblasts were not seen, it was labelled as myxoid sarcoma

The smears from the case of extraskeletal myxoid chondrosarcoma showed abundant extracellular myxoid material. (Fig. 1A) with embedded polygonal cells which did not show pleomorphism. Hence, FNAC diagnosis was given as Low grade myxoid sarcoma. (Fig. 1B)

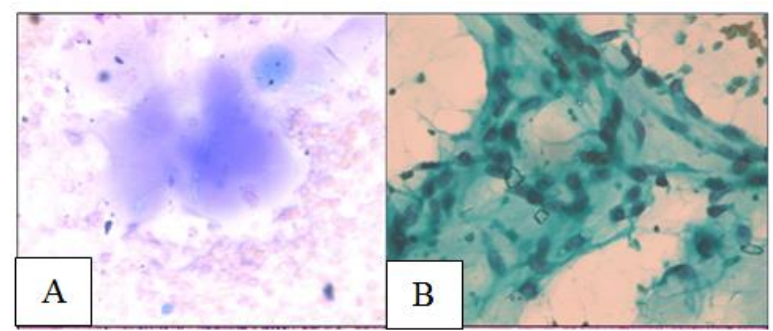

Fig. 1: Extra skeletal myxoid chondrosarcoma, 1A) Extracellular myxoid material. (MGG 100X), 1B) Polygonal to spindle cells embedded in the myxoid material. (PAP 400X)

Table 1: Myxoid Category $(n=4)$

\begin{tabular}{|l|c|c|c|c|}
\hline $\begin{array}{l}\text { No of cases with } \\
\text { HP Correlation }\end{array}$ & Age/sex & Location & Cytologic Diagnosis & Histologic Diagnosis \\
\hline 1 & $55 y e a r s / m a l e$ & Retroperitoneum & Myxoid Liposarcoma & Myxoid Liposarcoma \\
\hline 1 & 65 years/male & Perineal mass & Myxoid Sarcoma & Myxofibrosarcoma \\
\hline 1 & 52 years/male & Gluteal region & $\begin{array}{c}\text { Low grade } \\
\text { Myxoid sarcoma }\end{array}$ & $\begin{array}{c}\text { Extra skeletal Myxoid } \\
\text { chondrosarcoma }\end{array}$ \\
\hline 1 & 58 years/male & Neck & Chordoma (metastasis) & Chordoma (metastasis) \\
\hline
\end{tabular}

The patient with chordoma had a previous history of excision of chordoma of nasopharynx and now presented with a soft tissue nodule in the neck. The smears showed the characteristic physalliferous cells. (Fig. 2A \& 2B)

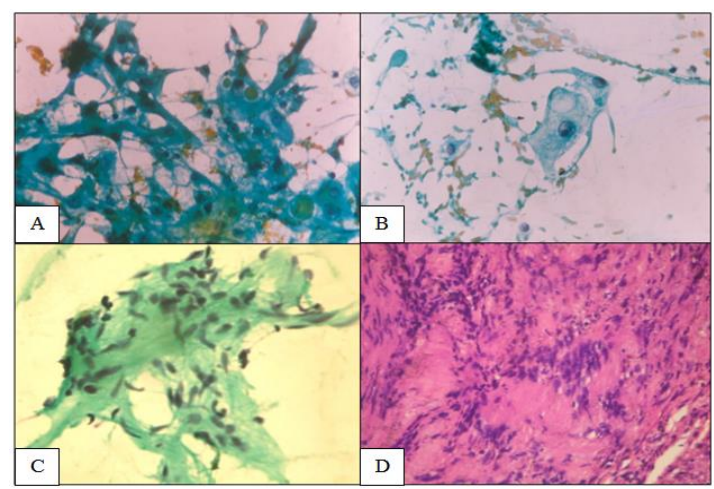

Fig. 2: A) Smear from chordoma. (PAP 100X), 2B)Physalliferous cell. (PAP 400X),

2C) Schwannoma-loosely arranged spindle cells separated by loose myxoid stroma. (PAP 100X), 2D) Schwannoma showing classical verocay bodies. (H \& E 100X)
The typing of spindle cell tumors into benign and malignant spindle cell sarcomas could be done accurately as shown in the table 2. $(n=20$. HP correlation available in 15) Aspirate in the case of schwannoma showed benign elongated slender nuclei with ill defined eosinophilic cytoplasm. (Fig. 2C,D) 
Table 2: Spindle cell category $(n=20$. HP correlation available in 15)

\begin{tabular}{|l|c|c|}
\hline $\begin{array}{l}\text { No. of cases with } \\
\text { HP Correlation }\end{array}$ & Cytologic Diagnosis & Histologic Diagnosis \\
\hline Benign & $\begin{array}{c}\text { Senign Spindle Cell Lesion (3), } \\
\text { Schwannoma (3) }\end{array}$ & Neurofibroma (4) \\
\hline 12 & $\begin{array}{c}\text { Benign Spindle Cell Lesion (2), } \\
\text { Neurofibroma (2) }\end{array}$ & Benign Fibrous Histiocytoma(1) \\
\hline & Benign spindle cell lesion (1) & Benign Fibrous Histiocytoma(1) \\
\hline & Benign Fibrous Histiocytoma(1) & Fibro Sarcoma (1) \\
\hline Malignant & High Grade Spindle Cell Sarcoma & $\begin{array}{c}\text { Malignant peripheral nerve sheath } \\
\text { tumor(MPNST) (1) }\end{array}$ \\
\hline 2 & High Grade Spindle Cell Sarcoma & Dermatofibrosarcoma protuberans(DFSP) (1) \\
\hline
\end{tabular}

Aspirate from neurofibroma showed loosely arranged, wavy spindle cells in a pale staining stroma. The grading of sarcomas was attempted on the basis of cellularity, nuclear overlapping and nuclear pleomorphism. Both the high grade sarcomas were of high grade on histology too.

But in the case of Dermatofibrosarcoma Protuberans (DFSP), which was a recurrent chest wall tumor in a 45 year old male, there was moderate cellularity, moderate pleomorphism and nuclear overlapping. The diagnosis was given as low grade sarcoma whereas DFSP is a sarcoma of intermediate grade.

No histopathological correlation was obtained in 5 cases, 3 cases were reported as benign spindle cell tumors and 2 cases as spindle cell sarcomas.

Table 3 depicts the distribution of the cases in pleomorphic category. $(n=2)$ Smears showed mostly pleomorphic tumor cells with multinucleated giant cells. By definition all pleomorphic sarcomas are regarded as high grade for therapeutic purposes.

The case of Malignant Peripheral Nerve Sheath Tumor (MPNST) was a recurrent forearm tumor in a 35 year old female with smears showing pleomorphic cells with multinucleated giant cells (Fig. 3A\& 3B). However the histology showed few areas with the characteristic features of MPNST (Fig. 3C). MPNST comes in the differential diagnosis of spindle cell tumors, this being a recurrent tumor presented as pleomorphic sarcoma. (Fig. 3D)

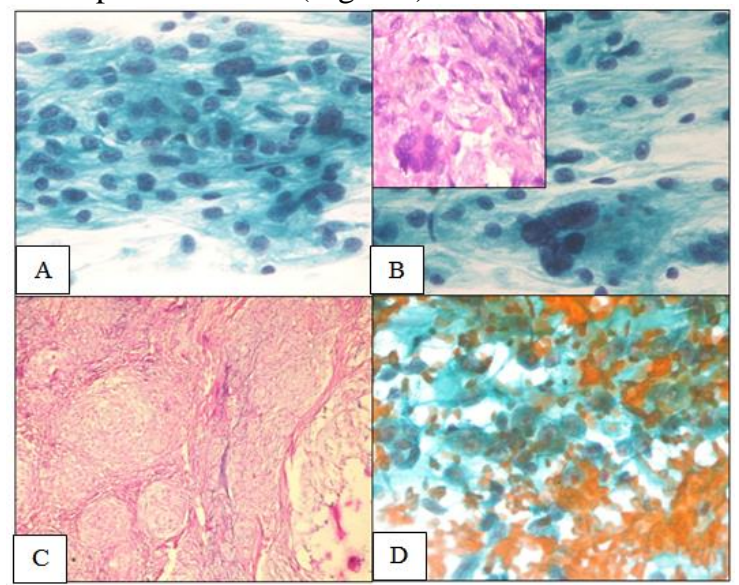

Fig. 3: Recurrent MPNST, 3A) Smear shows plump cellswith nuclear pleomorphism in a fibrillary background. (PAP400X), 3B) Bizarre multinucleated giant cell.(PAP 400X and $H \&$ E 400X), 3C) Monomorphic serpentine (spindle shaped cells) arranged in dense fascicles. (H \& E 100X), 4D) PLEOMORPHIC SARCOMA-cellular smears composed of polygonal cells with abundant cytoplasm, large vescicular nucleus and prominent nucleoli. (PAP 400X)

Table 3: Pleomorphic Category $(n=2)$

\begin{tabular}{|l|c|c|c|}
\hline $\begin{array}{c}\text { No of cases } \\
\text { with HP } \\
\text { correlation }\end{array}$ & Age/sex & Cytologic diagnosis & Histologic diagnosis \\
\hline 1 & 51 years/male & Pleomorphic sarcoma & MFH(Malignant Fibrous Histiocytoma) \\
\hline 1 & 35 years/male & Pleomorphic sarcoma & $\begin{array}{c}\text { Recurrent MPNST(Malignant Peripheral } \\
\text { Nerve Sheath Tumor) }\end{array}$ \\
\hline
\end{tabular}

Table 4 depicts the tumor from small round cell category $(n=4)$ Smears from the round cell category were highly cellular and composed of dyscohesive small round homogenous malignant cells with high $\mathrm{N}: \mathrm{C}$ ratio. Embryonal RMS showed small round cells which were admixed with spindle to polygonal, multinucleated tumor cells and rhabdomyoblasts which were diagnostic. 
Smears from a case of neuroblastoma showed well formed rosettes (Fig. 4A) on the CT guided FNAC smears, hence we called it as Neuroblastoma which was later confirmed on biopsy.

In the case of Ganglioneuroblastoma, which was a retroperitoneal mass, the FNAC diagnosis was offered as small round cell tumor. After the histopathological diagnosis, review of the smears showed occasional ganglion cells (Fig. 4B).

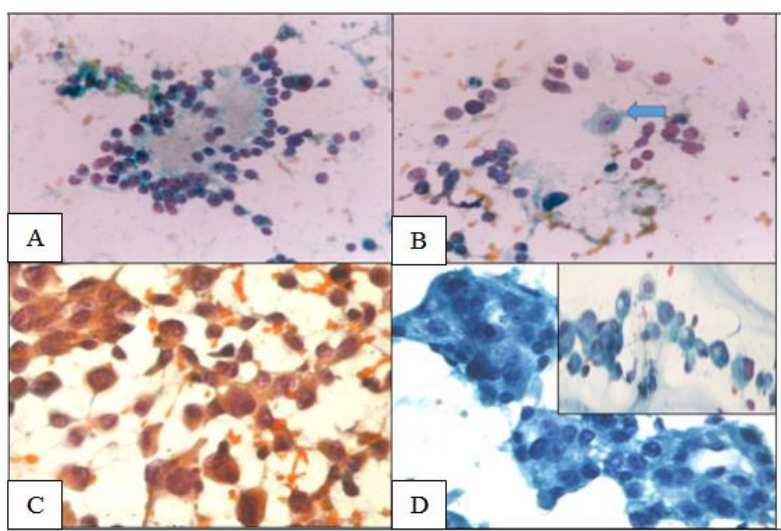

Fig. 4: 4A)Neuroblastoma -rosettes. (PAP 100X), 4B) Ganglioneuroblastoma showing ganglion cell (arrow). (PAP 100X), 4C) Metastatic melanoma showing pleomorphic polygonal cells with prominent nucleoli. (PAP 400X), 4D) Mets of adenocarcinoma showing glandular pattern and inset showing signet ring cells. (PAP 400X)

Table 4: Small round cell category $(n=4)$

\begin{tabular}{|l|c|c|c|c|}
\hline $\begin{array}{l}\text { No of cases } \\
\text { with HP } \\
\text { Correlation }\end{array}$ & Age/sex & Location & Cytologic diagnosis & Histologic diagnosis \\
\hline 1 & $3 y e a r s / f e m a l e$ & Vagina & $\begin{array}{c}\text { RMS } \\
\text { (Rhabdomyosarcoma) }\end{array}$ & $\begin{array}{c}\text { Embryonal RMS } \\
\text { (Rhabdomyosarcoma) }\end{array}$ \\
\hline 1 & 1 months/male & Retroperitoneum & Neuroblastoma & Neuroblastoma \\
\hline 1 & 7 month/male & Retroperitoneum & Small Round Cell tumor & Ganglioneuroblastoma \\
\hline 1 & $55 y e a r s / m a l e$ & Axilla & $\begin{array}{c}\text { Metastasis of } \\
\text { Lymphoma }\end{array}$ & $\begin{array}{c}\text { Metastasis of } \\
\text { Lymphoma }\end{array}$ \\
\hline
\end{tabular}

A 55 years old male who was an operated case of primary testicular diffuse large B cell lymphoma, presented with a diffuse soft tissue swelling in the axillary region. Smears were cellular, composed of round cells and occasional lymphoglandular bodies. In this case, the history helped in giving the diagnosis of metastasis of lymphoma in the soft tissue.

Smears from epithelioid/polygonal cell category $(n=7)$ category show dyscohesive polygonal tumor cells. The tumors in this category are epithelioid sarcoma, alveolar soft part sarcoma, malignant fibrous histiocytoma and epithelioid variants of leiomyosarcoma, MPNST, angiosarcoma etc.

Metastasis from epithelial malignancy and malignant melanoma are the main differentials to be considered in this category.

We did not have any case of primary epithelioid cell sarcoma, all the 7 cases were of metastasis: 2 from melanoma (Fig. 4C) presenting as subcutaneous nodules on thigh, 2 from carcinoma lung, presenting as subcutaneous nodules over forearm and chest walland 3 from carcinoma colon. (Fig. 4D), presenting as subcutaneous nodules over abdomen and chest wall.

We could type both the cases of metastatic melanoma on FNAC. In the other 5 cases, a differential diagnosis was offered in 2 cases as epithelial malignancy vs epithelioid sarcoma.

Presence of signet ring cells and glandular pattern in 3 cases helped to offer the diagnosis of metastatic carcinoma.

All the cases in lipomatous category $(n=108)$ were benign-Lipoma. Aspirates showed the characteristic fibrofatty fragments and mature adipocytes. Out of 108 cases, we received excision of 78 tumors and all were confirmed as Lipoma on histology.

Tumors not belonging to any of the above categories were included in the miscellaneous category. We had 7 cases of giant cell tumor of soft tissue and tendon sheath, which were predominantly located over fingers and foot, which were subsequently confirmed on histopathology. The aspirates showed the characteristic osteoclast like giant cells and xanthoma cells

We also had 18 cases which were non neoplastic non neoplastic soft tissue lesions. Aspirates from 2 subcutaneous nodules in the forearm showed microfilariae. (Fig. 5A)

Smears from a case of actinomycosis showed mixed inflammatory infiltrate and clumps of filamentous organisms. (Fig. 5B) 
40 years old female presented with a nodule over the abdominal hysterectomy scar, aspirates showed sheets of benign endometrial cells as well as the stroma. (Fig. 5 C\& 5D), Hence the diagnosis of scar endometriosis was given.

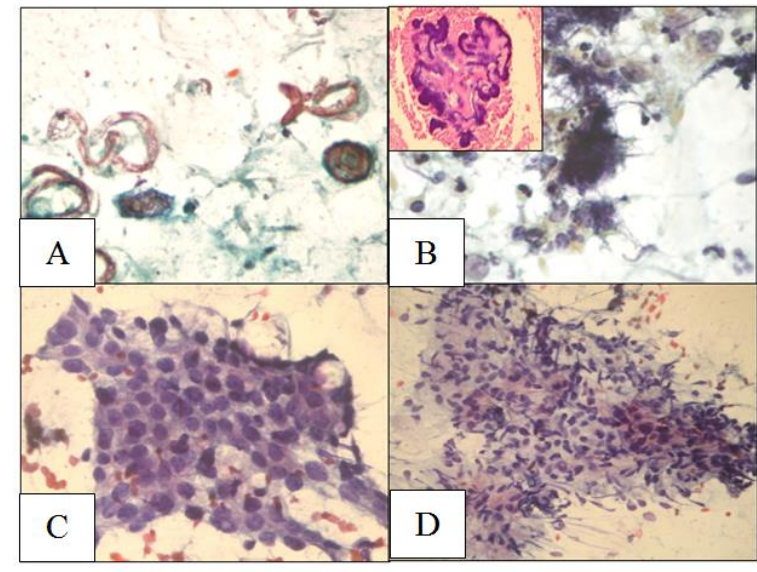

Fig. 5: 5A) Microfilariae.(PAP 100X), 5B)

Actinomycosis. (PAP 100X). Inset shows "sun ray appearance" of actinomycosis. (H \& E 100X), 5C) Endometrial cells in monolayered sheets. (PAP 400X), 5D) Stromal cells. (PAP 100X)

Table 5:

\begin{tabular}{|l|c|c|c|c|}
\hline Sr. No. & Studies & $\begin{array}{c}\text { Overall } \\
\text { Accuracy }\end{array}$ & $\begin{array}{c}\text { Accuracy to detect } \\
\text { benign lesions }\end{array}$ & $\begin{array}{c}\text { Accuracy to detect } \\
\text { malignant lesion }\end{array}$ \\
\hline 1. & Zubaida et $\mathrm{al}^{[3]}$ & - & $94 \%$ & $100 \%$ \\
\hline 2. & Parajuli et $\mathrm{al}^{[5]}$ & $80 \%$ & - & - \\
\hline 3. & Kulkarni et $\mathrm{al}^{[8]}$ & $93.75 \%$ & $93.33 \%$ & $93.93 \%$ \\
\hline
\end{tabular}

There were 6 cases of calcinosis cutis which were subcutaneous masses predominantly in gluteal region seen in elderly patients. The aspirate yielded chalky white material, laminated concretions and variable number of histiocytic lymphocytes and osteoclast like giant cells.

Diagnosis of tuberculous inflammation (cold abscess) was offered in 8 cases which were predominantly seen in chest wall region. Smears showed caseous necrosis with epithelioid cell conglomerates.

\section{Discussion}

Majority of our cases were benign (76.47\%). This was comparable to the studies by Zubaida et $\mathrm{al}^{3}{ }^{3}$ (89 $\%)$, Tailor et $\mathrm{al}^{4}(93.58 \%)$, Parajuli et $\mathrm{al}^{5}(80 \%)$. However, Rekhi et al, ${ }^{6}$ Dey et al, ${ }^{7}$ Kulkarni et al, ${ }^{8}$ observed predominance of malignant tumors.

Within the different neoplastic categories, benign tumors formed the majority in lipomatous category $(71.05 \%)$ and spindle cell category $(9.86 \%)$. While myxoid, epithelioid/polygonal and round cell tumors were predominantly malignant.

Rekhi et $\mathrm{al}^{6}$ observed (71.42\%) benign tumors in lipomatous category, all polygonal and myxoid were malignant. In the spindle cell category also majority were malignant. This was attributed to the fact that majority of their cases were referral sarcomas.

Regarding exact subtyping of tumors (with reference to histopathology), we could subtype all lipomas and miscellaneous category tumors and most of the round cell tumors, epithelioid/polygonal and myxoid tumors. Pleomorphic sarcomas could not be categorized correctly. In many cases correlation with history and location of tumor helped in diagnosis.

Rekhi et $\mathrm{al}^{6}$ could offer exact subtyping in all lipomatous and $70 \%$ pleomorphic tumors.

We obtained $50 \quad \%$ cytohistopathological correlation of sarcomas, Rekhi et $\mathrm{al}^{6}$ had higher rates.

Use of Immunohistochemistry improves the cytohisto concordance rates in sarcomas.

FNAC proved to be very useful in distinguishing non-neoplastic from neoplastic lesions. These findings have been endorsed by most of the authors ${ }^{4-6} \mathrm{We}$ could diagnose 11 cases of infections and FNAC was helpful in deciding further management.

FNAC also helped in detecting metastasis of carcinoma/melanoma in soft tissue.

We had 7 cases of metastasis. In 3 of these cases FNAC was the initial tool of investigation and subsequent workup helped in detecting the primary. Kulkarni et $\mathrm{al}^{8}$ noted $100 \%$ diagnostic accuracy in detecting metastasis.

We got $93.81 \%$ accuracy in cyto-histo correlation of the benign tumors and also 100\% accuracy in 
differentiating benign and malignant lesions. Our accuracy in exact subtyping of sarcomas was $50 \%$.

The table 5 compares the accuracy in benign and malignant lesions and the overall accuracy rates in other studies.

\section{Conclusion}

FNAC proved to be helpful in distinguishing between neoplastic and non-neoplastic soft tissue lesions, differentiating metastatic carcinoma and melanoma in soft tissue from primary soft tissue tumors and differentiating benign and malignant soft tissue tumors. We got $93.81 \%$ accuracy in cyto-histo correlation of the benign tumors and also $100 \%$ accuracy in differentiating benign and malignant lesions. Our accuracy in exact subtyping of sarcomas was $50 \%$.

FNAC is safe, easy, cost efficient primary tool in the diagnosis of neoplastic and non-neoplastic soft tissue lesions.

\section{References}

1. Barth RJ Jr, Merino MJ, Solomon D, Yang JC, Baker AR. A prospective study of the value of core needle biopsy and fine needle aspiration in the diagnosis of soft tissue masses. Surgery. 1992;112:536-43.

2. Chang AE, Matory YL, Dwyer AJ, Hill SC, Girton ME, Steinberg SM, et al. Magnetic resonance imaging versus computed tomography in the evaluation of soft tissue tumors of the extremities. Ann Surg. 1987;205:340-8.

3. Zubaida $\mathrm{R}$ et al. Utility of fine needle aspiration cytology in diagnosis of soft tissue lesions with histopathological correlation. Global journal of medicine and public health. 2013;2(2):1-7.

4. Tailor H J, Bhagat V M, Kaptan K B, Italiya S L, Balar H R, Agarwal M P: Diagnostic accuracy of fine needle aspiration aspiration cytology in soft tissue tumors: our institutional experience. International journal of research in medical sciences. 2013;1(4):443-447.

5. Parajuli S, Lakhey M. Efficacy of fine needle aspiration cytology in diagnosing soft tissue tumors. Journal of Pathology of Nepal. 2012;2:305-308.

6. Rekhi B, Gorad B D, Kakade A C, Shinoy R F. Scope of FNAC in the diagnosis of soft tissue tumors-A study from a tertiary cancer referral centre in India. Cytojournal. 2007;4(31):20.

7. Dey P, Mallik M K, Gupta S K, Vasishta R K. Role of fine needle aspiration cytology in the diagnosis of soft tissue tumours and tumour-like lesions. Cytopathology. 2004;15(1):32-7.

8. Kulkarni R D, Kokandakar H R, Kumbhakarna H R, Bhople K S. Fine needle aspiration cytology of soft tissue tumors in correlation with histopathology. Indian J. Pathol. Microbiology 2002;45(1):45-48. 